\title{
Quantum Mechanical/Molecular Mechanical (QM/MM) Car-Parrinello Simulations in Excited States
}

\author{
Marc-Etienne Moret, Enrico Tapavicza, Leonardo Guidoni, Ute F. Röhrig, Marialore Sulpizi, \\ Ivano Tavernelli, and Ursula Rothlisberger ${ }^{\star}$
}

\begin{abstract}
The combination of time-dependent density functional theory (TDDFT) for the description of excited states with a hybrid quantum mechanics/molecular mechanics (QM/MM) approach enables the study of photochemical processes in complex environments. Here, we present a short overview of recent applications of TDDFT/ MM approaches to a variety of systems including studies of the optical properties of prototypical organic and inorganic molecules in gas phase and solution, photoinduced electron transfer reactions in donor-bridge-acceptor complexes, and in situ investigations of the molecular mechanisms of photoactive proteins. The application of TDDFT/MM techniques to a wide range of systems enables an assessment of the current performance and limitations of these methods for the characterization of photochemical processes in complex systems.
\end{abstract}

Keywords: Car-Parrinello first-principles molecular dynamics · Excited states $\cdot$ Photoactive proteins $\cdot$ QM/MM simulations · Time-dependent density functional theory

\section{Introduction}

In the last years, time-dependent density functional theory (TDDFT) [1][2] has become widely used for the calculation of vertical excitation energies and excited state properties of medium to large size molecular systems. More recently, also excited state gradients and forces have been implemented in different quantum chemistry packages [3]. This feature is of special interest, because it allows the simulation of photochemical reactions and fluorescence spectra.
Here, we review some recent application of TDDFT combined with a hybrid QM/MM molecular dynamics (MD) scheme [4] for the inclusion of environmental effects.

After a short introduction of the methods, we present results for a variety of systems. Starting with the description of the cis-trans photoisomerization of simple prototypical molecules in gas-phase $(2,4-$ pentadiene-1-iminium cation and formaldimine), we then proceed to the study of excited state solution properties of the organometallic complex Ru-tris 2,2'-bipyridine and the photoinduced electron transfer in donor-bridge-acceptor molecules. As a final example, we will present results for the characterization of the molecular mechanisms governing light detection in the photoactive proteins PYP (Photoactive Yellow Protein) and bovine rhodopsin.

\section{Methods}

\subsection{Time-dependent Density Functional Theory (TDDFT)}

The response of a system to an external perturbation can be successfully studied within the linear density response (LR) approximation. The basic quantity in LRTDDFT is the density-density response function [5]

$$
\chi\left(\mathbf{r}, t, \mathbf{r}^{\prime}, t^{\prime}\right)=\left.\frac{\delta n(\mathbf{r}, t)}{\delta v_{e x t}\left(\mathbf{r}^{\prime}, t^{\prime}\right)}\right|_{v_{0}}
$$

which relates the first order density response $n_{1}(\mathbf{r}, t)$ to the applied perturbation $v_{1}(\mathbf{r}, t)$

$n_{1}(\mathbf{r}, t)=\int d^{3} \mathbf{r}^{\prime} d t^{\prime} \chi\left(\mathbf{r}, t, \mathbf{r}^{\prime}, t^{\prime}\right) v_{1}(\mathbf{r}, t)$

where $v_{0}(\mathbf{r})$ is the ground state KS potential and $v_{e x t}(\mathbf{r})=v_{0}(\mathbf{r})+v_{1}(\mathbf{r})$.

The response function for the physical system of interacting electrons, $\chi\left(\mathbf{r}, t, \mathbf{r}^{\prime}, t^{\prime}\right)$, can be related to the computationally more advantageous Kohn-Sham (KS) response, $\chi_{S}\left(\mathbf{r}, t, \mathbf{r}^{\prime}, t^{\prime}\right)$, and the problem of finding excitation energies of the interacting system reduces to the search for the poles of the response function.

In addition to the computation of excited state properties of a molecular system (excitation energies, densities and related properties), LR-TDDFT also enables the calculation of nuclear forces in the excited state. In the framework of the Lagrangian method [6], these forces are computed as derivatives of the total energy of the excited state with respect to the nuclear positions. 
The implementation of excited state forces within an MD package [3] allows therefore the efficient calculation of trajectories on an excited state surface at a modest computational cost. To account for non-adiabatic effects, a surface hopping algorithm, based on Landau-Zener [7] theory, was implemented [8]. In this approach, the probability of changing adiabatic state is calculated from the energy gap between the two adiabatic PES and the derivatives of the PES with respect to the reaction coordinate.

\subsection{Molecular Dynamics and $Q M / M M$}

In this study, we use two different QM/ MM excited state MD schemes based either on LR- TDDFT or a time propagation technique (P-TDDFT).

OurLR-TDDFT MD simulations are purely adiabatic and proceed in the following way. First a simulation in $S_{0}$ is carried out in order to obtain an equilibrated system at the target temperature of $300 \mathrm{~K}$ using a Nosé-Hoover thermostat. We then take random configurations (ionic coordinates and velocities) from this simulation and vertically excite the system into a selected singlet state. The positions of the nuclei are updated according to the forces computed as the gradients of the excited state energy. In a second iteration the ground state density and KS orbitals for the latest geometry are computed, and the linear response calculation provides the new excited state energy and the new forces on the ions.

This approach allows to follow adiabatically the instantaneous electronic excited state surface in a Born-Oppenheimer (BO) MD scheme. For all LR-TDDFT MD simulations, the Tamm-Dancoff approximation (TDA) [9] to TDDFT is applied.

Chemical reactions occur mostly in complex environments such as solutions, protein matrices or membranes. The interaction of the molecule of interest with the surrounding can be treated at different levels of theory. The method that we adopt here is based on the partitioning of the system into a chemically active site (computed at the DFT or TDDFT level) and a classically described environment. In the hybrid QM/MM MD approach, the total energy of a QM/MM system can be written as [4]

$$
E=E_{Q M}+E_{M M}+E_{Q M / M M}
$$

which correspond to the lowest eigenvalue of the Hamiltonian

$$
H=H_{Q M}+H_{M M}+H_{Q M / M M}
$$

where $H_{O M / M M}$ describes the interaction between the QM and the MM part. $H_{Q M / M M}$, the coupling Hamiltonian, can be divided into a bonded term, if covalent bonds exist between the QM and the MM subsystems, and a non-bonded term

$$
H_{\text {nonbonded }}=\sum_{i \in M M} q_{i} \int d \mathbf{r} \frac{\rho(\mathbf{r})}{\left|\mathbf{r}-\mathbf{R}_{i}\right|}+\sum_{\substack{i \in M M \\ j \in Q M}} v_{v d w}\left(R_{i j}\right)
$$

where $\mathbf{R}_{i}$ is the position of MM atom $i$ with the point charge $q_{i}, \rho$ is the total charge density of the quantum system (electronic and ionic), and $v_{v d w}\left(R_{i j}\right)$ is the van der Waals interaction between atoms $i$ and $j$. In the employed QM/MM scheme, the vanderWaals interaction term is simply taken from the classical force field. The Coulomb term is described at the QM level by replacing the classical point charge of the MM atoms by a suitable (smoother) charge distribution. Computational efficiency is achieved by modeling the long-range electrostatics by a Hamiltonian term that couples the multipole moments of the quantum charge distribution with the classical point charges. For details of the implementation see [10]. Alternatively, electrostatic interactions with the intermediate MM atoms can be taken into account by a scheme based on dynamically restrained electrostatic potential (D-RESP) charges [11]. If the QM/MM boundary cuts through a covalent bond, care has to be taken to saturate the valence orbitals of the QM system. In the present implementation, this can be done by "capping' the QM site with a hydrogen atom or an empirically parametrized pseudopotential [12].

\subsection{Computational Details}

All MD simulations are carried out with the CPMD code [13]. We use soft normconserving non-local Troullier-Martins pseudopotentials [14] and a 70 Ry energy cutoff for the plane wave expansion of the wave functions. The inherent periodicity in the plane wave calculations is circumvented solving Poisson's equation for non-periodic boundary conditions. The BLYP [15] and the PBE [16] functionals are employed.

The CPMD implementation of LRTDDFT makes use of the Tamm-Dancoff approximation (TDA) [3]. All energies and gradients are converged to a maximum deviation of $10^{-7}$ a.u. A time integration step of 5 to 15 a.u. (0.12 to $0.36 \mathrm{fs}$ ) is used. Oscillator strengths are computed according to Bernasconi et al. [17]. Ground state simulations are carried out within the Car-Parrinello MD algorithm [18], while excited state simulations follow the $\mathrm{BO}$ MD scheme. Since this framework treats the nuclei as classical particles, we cannot capture the quantized vibrational structure of the spectra and the Franck-Condon factors.

\section{Applications}

\subsection{Photoinduced Isomerization in Gas Phase Model Compounds}

Photoinduced cis-trans isomerizations of $\mathrm{C}=\mathrm{C}$ and $\mathrm{C}=\mathrm{N}$ double bonds are investigated in two model compounds, namely 2,4-pentadiene-1-iminium cation (PSB) and formaldimine (Fig. 1) [19].

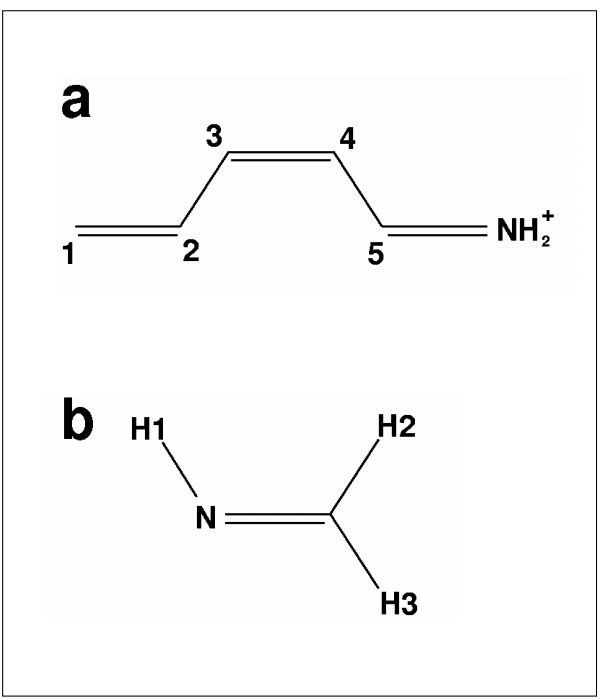

Fig. 1. (a) 2,4-pentadiene-1-iminium cation (protonated shiff base, PSB); (b) formaldimine.

If no intersystem crossing to a triplet surface occurs, the reaction path of photochemical reactions of organic molecules is determined primarily by the topology of the excited singlet surfaces $S_{x}$, along with the topology of the ground state $\mathrm{S}_{0}$.

Photochemical cis-trans isomerizations play an important role in many biological and chemical processes. These reactions occur on excited state surfaces characterized by potential energy minima at geometries which are particularly unfavorable in $\mathrm{S}_{0}\left(\sim 90^{\circ}\right.$ bond twist $)$. At these 'biradicaloid geometries' the double bond is essentially reduced to a single bond. The planar ground state structure is unfavorable in the excited state because one of the two unpaired electrons occupies an antibonding orbital, and this destabilization dominates the energy balance.

In some cases, the excited trajectory can reach a conical intersection between $S_{1}$ and $\mathrm{S}_{0}$, followed by a rapid radiationless transition to the ground state and a fast relaxation along the $\mathrm{S}_{0}$ potential energy surface (PES). The final product of a photoisomerization can either be the same isomer as initially excited or the opposite one, depending on the dynamical memory conserved during the transition. 


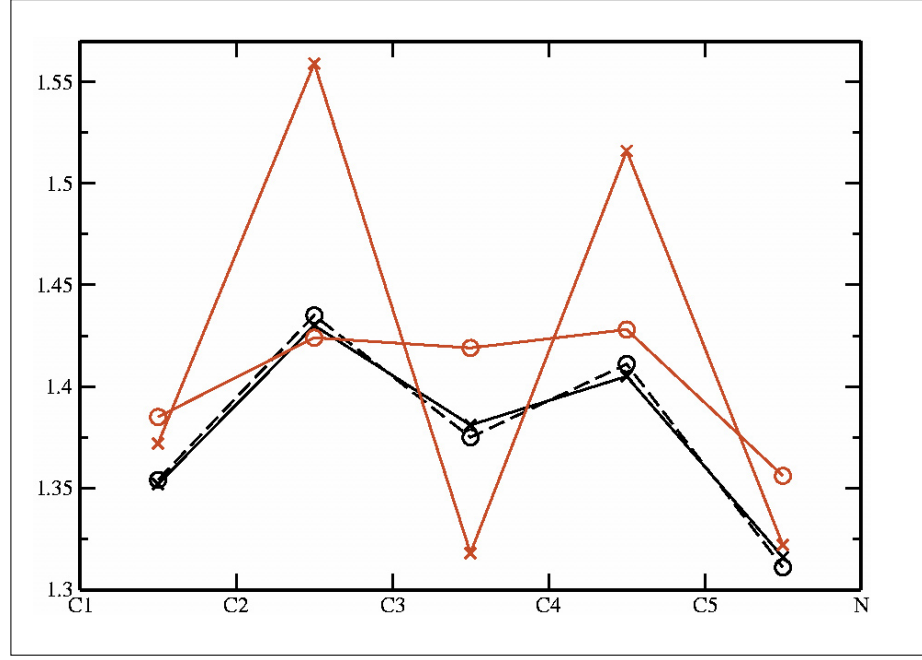

Fig. 2. $P S B$ bond length in planar optimized $\mathrm{S}_{0}$ (black) and $\mathrm{S}_{1}$ (red) structures. TDDFT/PBE/TDA values are shown with crosses, CASPT2 values from [38] are shown with circles.

An advantage of the MD approach consists in the completely unconstrained relaxation of the system on the (3N-6)-dimensional PES spanned by the internal degrees of freedom of the molecule. This yields the possibility to study the trajectory following photoexcitation and to identify the most important degrees of freedom involved in the relaxation in an unbiased way. The projection of a trajectory into the one-dimensional or two-dimensional space of the relevant degrees of freedom is performed here only for analysis and visualization purposes.

\subsubsection{Protonated Schiff Base}

The 2,4-pentadiene-1-iminium cation (PSB, see Fig. 1a) is a theoretically wellinvestigated model system of the chromophore in the visual pigment rhodopsin. PSB does not possess any lone pair orbital, so all valence excited singlet states are of $\pi \rightarrow \pi^{*}$ character. CASSCF/CASPT2 calculations predict that in vacuum the spectroscopic state is $\mathrm{S}_{1}$, an ionic single HOMO-LUMO transition, while $S_{2}$, a covalent doubly excited state, is not involved. In $S_{1}$, the initial motion along the minimum energy path is dominated by stretching modes, leading to a planar stationary structure with decreased bond length alternation (elongated central double bond, see Fig. 2 ). After the 'turning point' $\left(\mathrm{d}_{\mathrm{C} 3-\mathrm{C} 4} \sim 1.53 \AA\right.$, $\left.\varphi_{\mathrm{C} 2-\mathrm{C} 3-\mathrm{C} 4-\mathrm{C} 5} \sim 25^{\circ}\right)$, the twisting motion becomes dominant, and the molecule undergoes a barrierless relaxation towards a conical intersection with $\mathrm{S}_{0}$ at a twist angle $\varphi_{\mathrm{C} 2-\mathrm{C} 3-\mathrm{C} 4-\mathrm{C} 5}$ of about $80^{\circ}$. During this process, a shift of electron density from the carbon end towards the Schiff base end is observed, which reaches its maximum at the conical intersection.

In contrast to the CASSCF/CASPT2 results, tight-binding and full TDDFT calculations of different protonated Schiff bases yield a stationary point on the $S_{1}$ surface

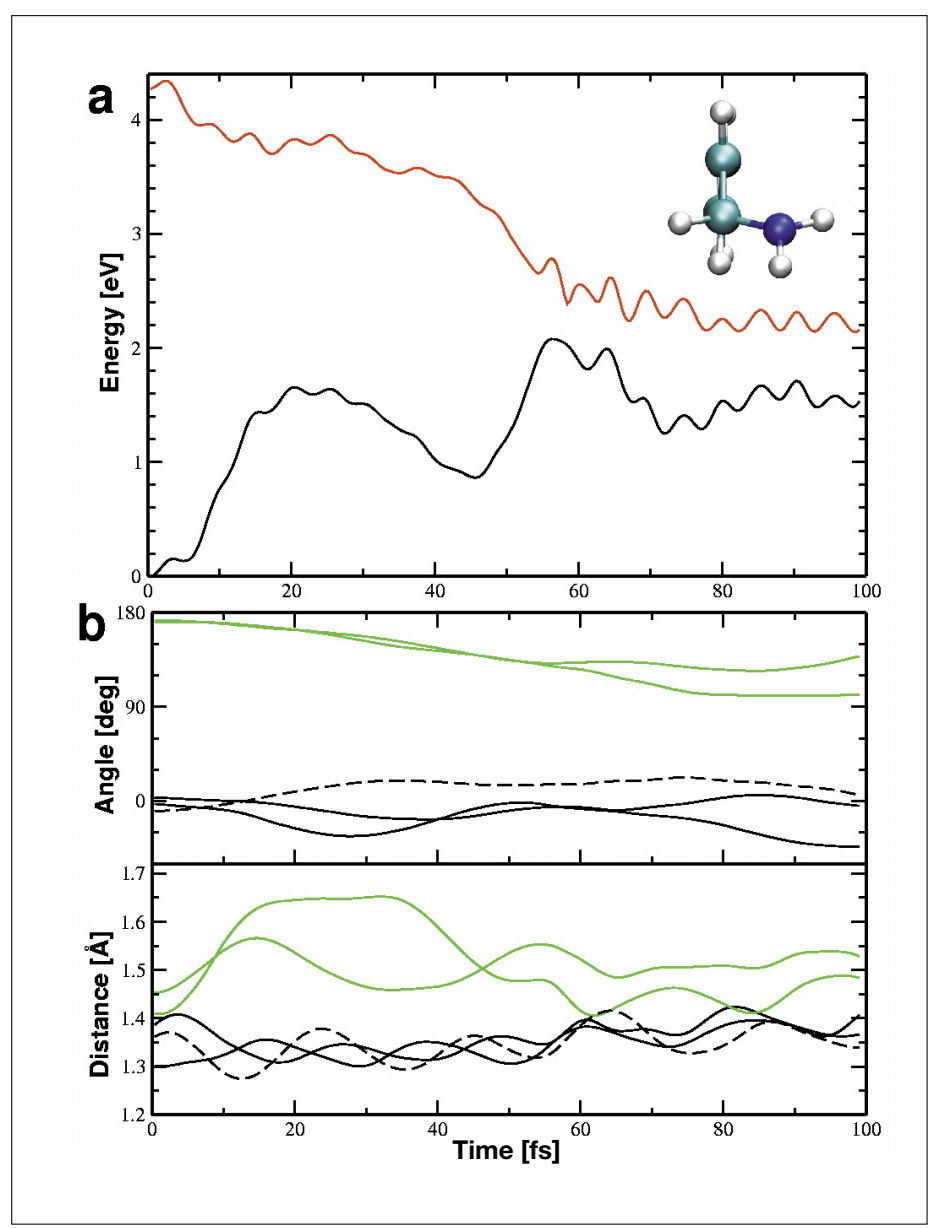

Fig. 3. LR-TDDFT simulation of PBS. (a) Time evolution of the $S_{2}$ energy (red) and the $S_{0}$ energy (black) along one $S_{1}$ simulation (temperature control $300 \pm 100 \mathrm{~K}$ ). Inset: optimized $S_{1}$ structure viewed along the $C_{3}-$ C4 bond. (b) Dihedral angles (black, around double bonds; green, around single bonds) and bond lengths (black, double bonds; green, single bonds) from the same simulation. The central dihedral angle $\theta_{\mathrm{C} 2-\mathrm{C} 3-\mathrm{C} 4-\mathrm{C} 5}$ and the central double bond $\mathrm{d}_{\mathrm{C} 3-\mathrm{C} 4}$ are shown as dashed lines.

with an increased bond length alternation, meaning that the double bonds become even shorter and rotation is strongly hindered. In addition, the charge distribution in $\mathrm{S}_{1}$ has been found to be nearly identical to that in $\mathrm{S}_{0}$ for all stationary points. It has been concluded that TDDFT yields an erroneous $S_{1}$ gradient and charge distribution, and the errors are ascribed to the local approximation of the exchange-correlation potential.

Multiple MD simulations are carried out in $\mathrm{S}_{0}$ and $\mathrm{S}_{1}$ states in gas phase. In $\mathrm{S}_{0}$ all dihedral angles fluctuate around 0 or $180^{\circ}$. Fig. 3 shows the time evolution of the $S_{1}$ and $S_{0}$ energies along one $S_{1}$ trajectory (temperature control $300 \pm 100 \mathrm{~K}$ ) using LR-TDDFT MD. A large decrease in the $\mathrm{S}_{1}$ energy by $2 \mathrm{eV}$ is observed at the same time as an increase of the $S_{0}$ energy of the same amount, but the two surfaces do not intersect. The $S_{1}$ relaxation is characterized by a large increase in the single bond lengths (see Fig. 3) and a rotation around these bonds $\left(\varphi_{\mathrm{C} 1-\mathrm{C} 2-\mathrm{C}_{3}-\mathrm{C} 4}=100^{\circ}\right)$. The final structure after $100 \mathrm{fs}$ looks similar to the optimized $S_{1}$ structure (see inset in Fig. 3).
We also performed TDDFT MD simulations using the Ehrenfest propagation scheme [19]. Here, we initially excited one electron from the HOMO to the LUMO. In this case, we find a remarkable difference between the LR-TDDFT and the P-TDDFT schemes: while LR-TDDFT decreases the double bond lengths, P-TDDFT increases them in agreement with CASPT2 and restricted open shell KS (ROKS) [20] calculations. The central double bond adopts an average length of $1.441 \AA$ in good agreement with the ROKS value. The time evolution of the dihedral angle $\varphi_{\mathrm{C} 2-\mathrm{C} 3-\mathrm{C} 4-\mathrm{C} 5}$ reaches $\sim 40^{\circ}$, but no complete isomerization is observed during the simulated time of 110 fs. Unfortunately, due to high computational costs of the Ehrenfest propagation and the intrinsic need to consider nonadiabatic effects in order to control the deviation from the BO surface, the trajectory presented here are limited to the first $140 \mathrm{fs}$ after excitation.

In summary, the LR-TDDFT results for PSB are in clear disagreement with CASSCF/CASPT2 results and the experi- 
mental detection of photoinduced double bond isomerizations in similar compounds. Although the vertical excitation energies for the ground state optimized structure are in good agreement between CASPT2 and TDDFT (if the dark state $S_{2}$ is neglected), the shape of the $S_{1}$ PES is different. This seems to concern mainly the bond length alternation, since it has been shown that TDDFT yields reasonable energies along the twisting coordinate. However, once the molecule is trapped in the local minimum characterized by short double bonds, the barrier towards isomerization is very high and cannot be overcome by its kinetic energy. Therefore MD simulations in $S_{1}$ with LR-TDDFT yield a single bond rotation, while CASSCF calculations yield a double bond isomerization. The P-TDDFT scheme on the other hand generates the correct bond length alternation and its trajectory leads to a (partial) rotation around the correct double bond (for a discussion see [19]).

\subsubsection{Formaldimine}

Formaldimine (methyleneimine) is very reactive and decomposes by polymerization, oxidation, or hydrolysis. It plays an important role as the smallest member of the large class of imines and in addition it is of astrophysical interest, having been detected in dark interstellar dust clouds. In the laboratory, $\mathrm{H}_{2} \mathrm{CNH}$ can only be observed transiently by pyrolysis of amines or different azido compounds, and its electronic absorption spectrum has only been recorded very recently. The spectrum shows a broad and structureless peak with a maximum at $250 \mathrm{~nm}(4.96 \mathrm{eV})$ in close agreement to our calculated vertical excitation energy of 4.92 $\mathrm{eV}$. After radiationless decay to the ground state the molecule possesses enough internal energy for fragmentation. The molecular structure of formaldimine in the ground state obtained by microwave spectroscopy [21] agrees very well with our calculated structure [19].

Theoretical investigations of the photoisomerization in formaldimine have found the twisting pathway to be preferred over the in-plane mechanism [22-24].

$\mathrm{H}_{2} \mathrm{CNH}$ contains five atoms and has therefore nine internal degrees of freedom that are all free to relax in the excited state MD simulations. For the analysis of the generated trajectories we will focus on the valence angle $\alpha_{\mathrm{CNH}}$, the dihedral angle $\varphi_{\mathrm{H} 3 \mathrm{CNH}}$ (for atom numbering see Fig. 1), and the pyramidalization angle of the carbon atom $\omega_{\mathrm{Pyr}}$, defined as the angle between the $\mathrm{H}-\mathrm{C}-\mathrm{H}$ plane and the $\mathrm{C}=\mathrm{N}$ bond. In the $\mathrm{S}_{1}$ simulations without any temperature control, an intersection between $\mathrm{S}_{1}$ and $\mathrm{S}_{0}$ is reached several times (Fig. 4).

The crossing point is characterized by the coordinates $\varphi_{\mathrm{H} 3 \mathrm{CNH}} \sim 100^{\circ}, \alpha_{\mathrm{HNC}}$ $\sim 100^{\circ}, \omega_{\mathrm{Pyr}} \sim 12^{\circ}$. This point is not located

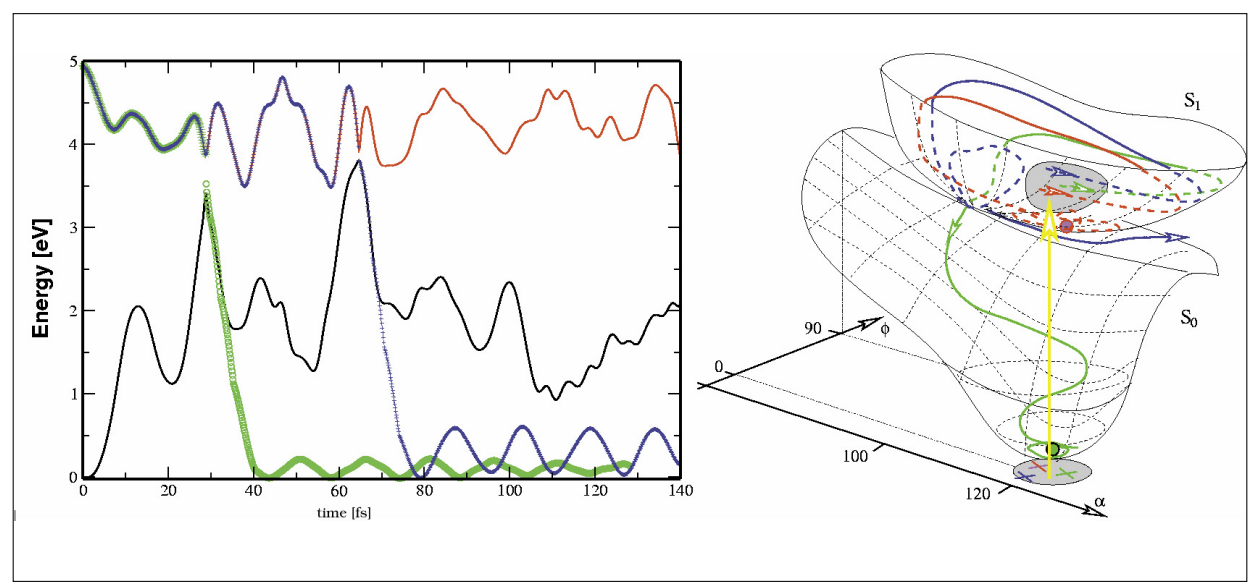

Fig. 4. LR-TDDFT simulation of formaldimine without temperature control. Time evolution of the $\mathrm{S}_{0}$ (black) and $\mathrm{S}_{1}$ (red) energies. Two different trajectories are shown in blue and green. On the right panel, qualitative PES diagram of the photoexcitation and relaxation processes.

at the minimum of the PES $\left(\varphi=101^{\circ}, \alpha=\right.$ $110^{\circ}, \omega=19^{\circ}$ ) and differs especially in the bonding angle $\alpha$. A detailed characterization of these regions of contact between the surfaces $\mathrm{S}_{0}$ and $\mathrm{S}_{1}$ is not in the scope of this work. All surface crossings observed in our simulations are characterized by large values for the derivative in time of the energy gap between the two adiabatic states which translate, according to Landau-Zener theory, into a high probability for a jump between the surfaces. For all crossings described in this section, the probabilities for the jump to $\mathrm{S}_{0}$ are larger than $99 \%$, leading to a radiationless transition. Different possibilities exist for the course of such non-adiabatic trajectories (see Fig. 4): the first trajectory (green) jumps from state $S_{1}$ to state $S_{0}$ at the first crossing point. Once in the ground state, the system relaxes rapidly towards the basin of attraction on the reactant side. For the case that the system remains on the excited state surface until the second surface crossing occurs (blue curve), the relaxation on $\mathrm{S}_{0}$ leads instead to the isomerized product. The time spent in $S_{1}$ between the two crossings ( $36 \mathrm{fs}$ ) corresponds to a full angle bending period and brings the system to the surface crossing conformation. The additional two curves (black and red) of Fig. 4a correspond to the energy time series for the excited and ground states respectively, when the forces driving the dynamics are computed from the $S_{1}$ surface for the full trajectory. In this case, after the first $100 \mathrm{fs}$ of relaxation, the system thermalizes to the minimum on the excited state PES.

\subsection{Ruthenium tris-2,2'-Bipyridine}

Ruthenium polypyridine compounds have received much attention in the last decades. One of their most prominent features is their ability to undergo metal-to-ligand charge-transfer (MLCT) transitions upon light absorption, generating highly reactive excited states that can easily transfer an electron to another species. This property has led to several applications, in both fundamental and applied contexts. As an example, the best suited dyes for photosensitized nanocrystalline semiconductor based solar cells belong to this class of compounds [25].

Ruthenium tris-2,2'-bipyridine (Fig. 5) can be seen as the prototype for these photoinduced MLCT transitions, and has been extensively studied via spectroscopic techniques. Its vertically excited singlet MLCT state is known to undergo fast conversion to a long lived ( $1 \mu$ s in water) triplet $\left({ }^{3} \mathrm{MLCT}\right)$ state [26], and the recent development of ultrafast X-ray spectroscopy techniques allows some structural characterization of this state in aqueous solution [27].

An accurate description of the photophysical properties of $\left[\mathrm{Ru}(\mathrm{bpy})_{3}\right]^{2+}$ is likely to require the inclusion of solvent effects, as dynamical measurements have been shown to be strongly solvent dependent [28]. Thus, QM/MM simulation techniques, in parallel with DFT and TDDFT calculations, are ideally suited for such a study.

First, a geometry optimization and a KS orbital analysis were performed in vacuo for

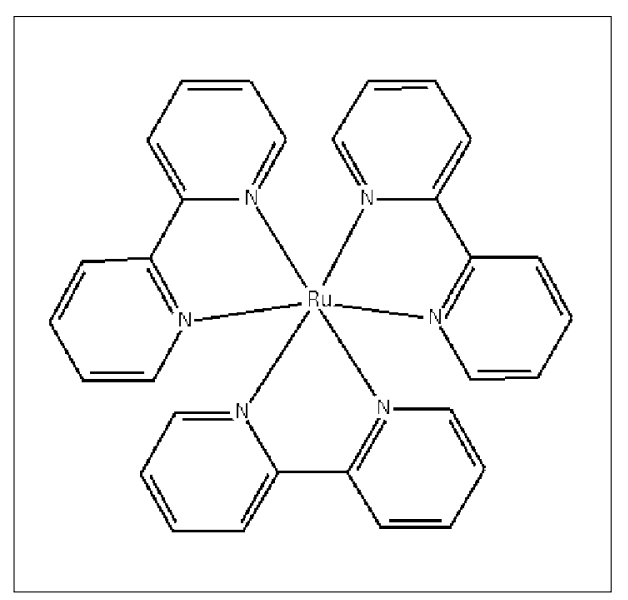

Fig. 5. Ruthenium tris-2,2'-bipyridine 
the ground state. These calculations showed few deviations from the X-ray crystal structure and from previous calculations by Daul et al. [29]. In particular, we found that the three highest occupied molecular orbitals (HOMOs) were metal centered $d$ orbitals, and the three lowest unoccupied molecular orbitals (LUMOs) were a set of linear combinations of ligand $\pi^{*}$ orbitals

A single point TDDFT calculation in vacuo showed that the first five singlet-singlet transitions were close to single electron transitions. The first transition, as well as the two transitions with a large oscillator strength, ended into fully delocalized, $D_{3}$ symmetric states. The absolute energy of the first strongly absorbing band $(\sim 2.18 \mathrm{eV})$ is in reasonable agreement with experiment $(2.67 \mathrm{eV})$ [30].

TDDFT calculations were then performed on instantaneous structures taken from a QM/MM trajectory at $300 \mathrm{~K}$ including explicit classical water molecules and chloride counter ions. With respect to the gas phase, the solution data showed a red shift of 0.1 to $0.3 \mathrm{eV}$ for the first transition and for the highly absorbing ones. This can be rationalized by the stabilization of the increasing charge on the $\mathrm{Ru}$ atom and the negative charge appearing on the ligands by water dipoles. This TDDFT study shows that the combined QM/MM - TDDFT scheme that has already been successfully applied to the optical properties of acetone and aminocoumarins in different solutions [31-33] is also applicable to transition metal complexes such as $\left[\mathrm{Ru}(\text { bpy })_{3}\right]^{2+}$ (Fig. 6, Fig. 7). However, although this work indicates a trend, it does not allow us to draw definitive conclusions. A more complete study including more states and a statistically significant number of structures would be necessary for a comprehensive understand-

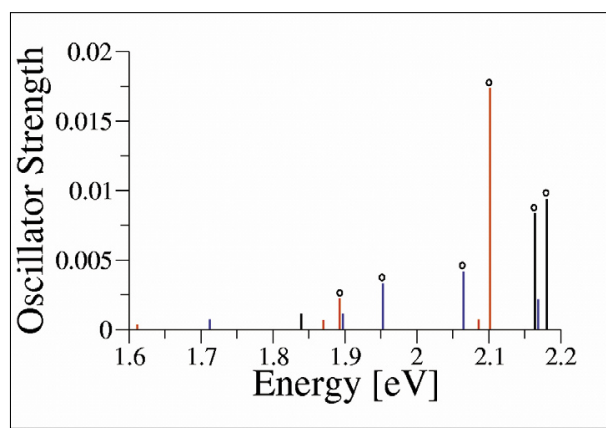

Fig. 7. Comparison between the excitation energies and oscillator strengths of the first five singlet-singlet transitions of $\left[\mathrm{Ru}(\mathrm{bpy})_{3}\right]^{2+}$ in vacuo (black) and from two snapshots from the QM/MM trajectory in water (red and blue). Circles indicate transitions mainly promoting an electron from the HOMO-1 and HOMO-2 orbitals to ligand based orbitals.

ing of light absorption by ruthenium polypyridine compounds.

\subsection{Electron Transfer in Covalently Linked Donor-Bridge-Acceptor Molecules}

Intramolecular photoinduced electron transfer (PET) in donor-bridge-acceptor (DBA) systems is one of the most extensively studied processes in chemistry. PET reactions are characterized by a spontaneous redistribution of electron density after an optical HOMO-LUMO excitation, where HOMO and LUMO are located both on the same photoactive site of the molecule: $\mathrm{D}$ $\mathrm{B}-\mathrm{A}+\mathrm{h} v \rightarrow \mathrm{D}^{*}-\mathrm{B}-\mathrm{A} \rightarrow \mathrm{D}^{+}-\mathrm{B}-\mathrm{A}^{-}$. Here, we investigate $\mathrm{PET}$ in a covalently linked organic DBA molecule (Fig. 8).

The electronic spectra of the system has been calculated using LR-TDDFT in combination with the GGA exchange correla- tion functionals PBE, BLYP and the hybrid functional B3LYP. Important for the PET is the experimental transition at $4.24 \mathrm{eV}$, corresponding to a $n-\pi^{*}$ excitation of the donor, typical for aniline derivatives. Symmetry considerations and the localization of the KS orbitals suggest to assign the $\mathrm{H} \rightarrow$ $\mathrm{L}+1$ transition to the $\mathrm{n}-\pi^{*}$ transition (Fig. $8)$. For this transition, all functionals employed here result in an accuracy of $0.3 \mathrm{eV}$ compared to the experimental value.

BO MD was performed to simulate the process of PET using excited state forces as implemented in CPMD [3]. As initial configuration the $4^{\text {th }}$ excited state, which corresponds to the $\mathrm{H} \rightarrow \mathrm{L}+1$ excitation was chosen. The evolution of the charge distribution was monitored by evaluating the electron density at each time step. In addition, the electronic spectra were calculated along the whole trajectory allowing the computation of the PES for the ground and excited states. In this way, the process of charge separation can be related to the occupation of the KS orbitals associated to the PES which drives the nuclear motion. Using the Landau-Zener surface hopping algorithm, it is possible to follow the reaction path from photoexcitation to charge separation. The evolution of the electron density of the molecular fragments defined as donor, bridge and acceptor moieties shows a net charge transfer of $\sim 1$ electron to the acceptor after about $120 \mathrm{fs}$. Geometrical analysis of the trajectory allows the identification of the modes coupled to the charge transfer event. The most relevant geometrical change can be related to the hybridization of the nitrogen atom at the donor site. During the charge transfer it changes from a tetrahedral to a planar geometry, corresponding to a change from $\mathrm{sp}^{3}$ to $\mathrm{sp}^{2}$ hybridization.

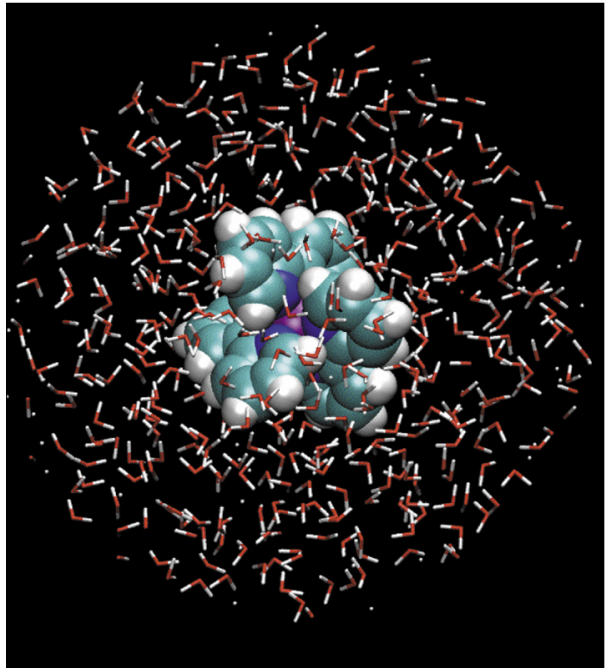

Fig. 6. A snapshot from the QM/MM simulation of $\left[\mathrm{Ru}(\mathrm{bpy})_{3}\right]^{2+}(\mathrm{QM})$ in water (MM). For clarity, water molecules and chloride ions beyond $15 \AA$ from the Ru atom have been omitted.

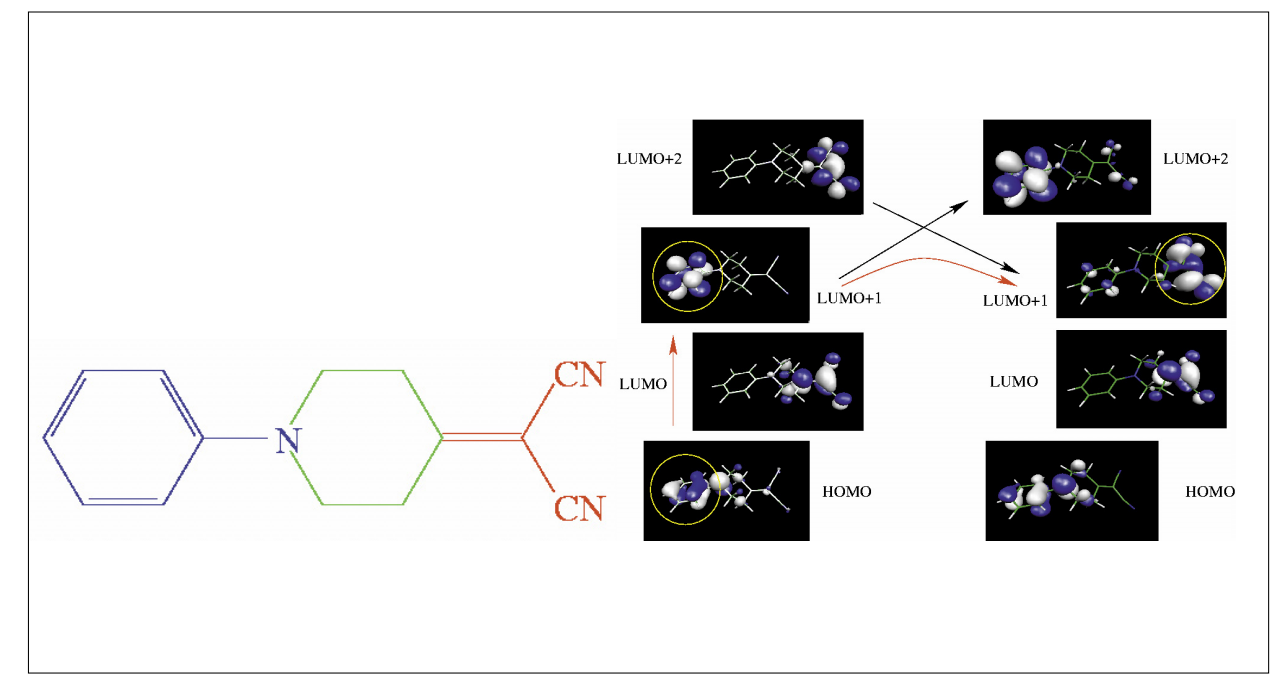

Fig. 8. Left: The investigated DBA molecule. The electron donor, bridge and electron acceptor are shown in blue, green and red respectively. Right: Kohn-Sham orbitals of the DBA1 molecule. The suggested mechanism for excitation and charge transfer is indicated. 


\subsection{Photoactive Proteins}

QM/MM Car-Parrinello simulations of excited states enable the in situ investigation of photochemical processes in biological systems. We are currently applying this technique to investigate the molecular mechanisms of bacterial and mammalian light detection in a parallel study of the two photoactive proteins PYP (Photoactive Yellow Protein) and bovine rhodopsin (Fig. 9). We have first characterized the properties of the two chromophores (Fig. 9) in solution as well as in the protein environment [34][35]. By using classical as well as QM/MM simulations we have also identified the molecular relaxation processes that follow photoexcitation in the time window from few femtoseconds to nanoseconds [36][37].

In the case of the visual pigment rhodopsin, we could demonstrate that the photoinduced cis-trans isomerization can take place with minute structural changes (RMSD of the chromophore of only 0.4 $\AA$ ) creating an internally strained molecule which relaxes on the nanosecond time scale by a switch of the ionone ring. In this process, the chromophore acts as a molecular spring that transfers the mechanical strain to the protein environment triggering predominantly changes in the $6^{\text {th }}$ transmembrane helix.

Received: May 19, 2005

[1] E. Runge, E.K.U. Gross, Phys. Rev. Lett. 1984, 53, 997.

[2] P. Hohenberg, W. Kohn, Phys. Rev. B 1964, 136, 864; W. Kohn, L.J. Sham, J. Phys. Rev. A 1965, 140, 1133.

[3] J. Hutter, J.Chem. Phys. 2003, 118, 3928.

[4] For recent reviews see: M.C. Colombo, L. Guidoni, A. Laio, A. Magistrato, P. Maurer, S. Piana, U. Röhrig, K. Spiegel, M. Sulpizi, J. VandeVondele, M. Zumstein, U. Rothlisberger, Chimia, 2002, 56, 11-17; P. Sherwood, in 'Modern Methods and Algorithms of Quantum Chemistry', Ed. J. Grotendorst, John von Neumann Institute for Computing, Jülich, NIC Series, 1, 2000, p. 257.

[5] R. Bauernschmitt, R. Ahlrichs, Chem. Phys. Lett. 1996, 256, 454; M. Petersilka, U.J. Gossmann, E.K.U. Gross, Phys. Rev. Lett. 1996, 76, 1212.

[6] D. Marx, J. Hutter, 'Modern Methods and Algorithms of Quantum Chemistry', Ed. J. Grotendorst, John von Neumann Institute for Computing, Jülich, NIC Series, 1, 2000, p. 301.

[7] L.D. Landau, Phys. Z. Sowjetunion 1932, 2, 46; C. Zener, Proc. R. Soc. A 1932, 137, 696.

[8] E. Tapavicza, I. Tavernelli, U. Rothlisberger, manuscript in preparation.

[9] S. Hirata, M. Head-Gordon, Chem. Phys. Lett. 1999, 314, 291.

[10] A. Laio, J. VandeVondele, U. Rothlisberger, J. Chem. Phys. 2002, 116, 6941; J. VandeVondele, U. Rothlisberger, J. Chem. Phys.
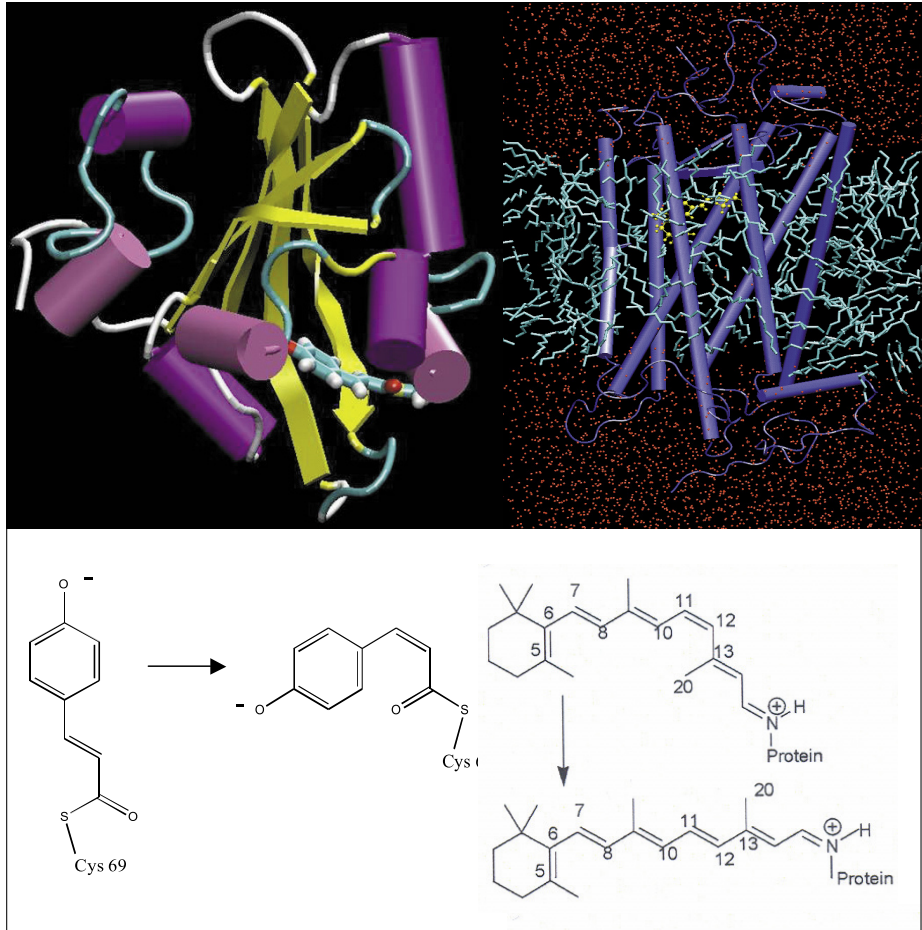

Fig. 9. Bacterial light detector PYP (upper left) and mammalian visual pigment rhodopsin (upper right). Chromophores are represented in a ball and sticks model. The chemical formula of the chromophores and the photoreaction are given below.

2000, 113, 4863; J. VandeVondele, U. Rothlisberger, J. Chem. Phys. 2001, 115, 7859.

[11] A. Laio, J. VandeVondele, U. Rothlisberger, J. Phys. Chem. B 2002, 106, 7300.

[12] O.A. von Lilienfeld, I. Tavernelli, U. Rothlisberger, D. Sebastiani, J. Chem. Phys. 2005, 122, 014113.

[13] CPMD, IBM Corp. (1990-2001). Copyright MPI für Festkörperforschung Stuttgart (1997-2001). Available online at: http://www.cpmd.org.

[14] N. Trouiller, J.L. Martins, Phys. Rev. B 1991, 43, 1993.

[15] A.D. Becke, Phys. Rev. A 1988, 38, 3098; C. Lee, W. Yang, R.G. Parr, Phys. Rev. B 1988, 37, 785.

[16] J.P. Perdew, K. Burke, M. Ernzerhof, Phys. Rev. Lett. 1996, 77, 3865.

[17] L. Bernasconi, M. Sprik, J. Hutter, $J$. Chem. Phys. 2003, 119, 12419.

[18] R. Car, M. Parrinello, Phys. Rev. Lett. 1985, 55, 2471.

[19] I. Tavernelli, U.F. Röhrig, U. Rothlisberger, Mol. Phys. 2005, 103, 963.

[20] I. Frank, J. Hutter, D. Marx, M. Parrinello, J. Chem. Phys. 1998, 108, 4060.

[21] R. Pearson Jr, F.J. Lovas, J. Chem. Phys. 1977, 66, 4149.

[22] V. Bonačić-Koutecky, M. Persico, J. Am. Chem. Soc. 1983, 105, 3388; V. BonačićKoutecky, J. Michl, Theor. Chim. Acta 1985, 68, 45.

[23] R. Sumathi, J. Molec. Struct. (Theorchem.) 1996, 364, 97

[24] I. Frank, J. Hutter, D. Marx, M. Parrinello, J. Chem. Phys. 1998, 108, 4060; N. L. Doltsinis, D. Marx, Phys. Rev. Lett. 2002, $88,166402$.
[25] M. Grätzel, Nature 2001, 414, 338-344; A. Hagfeldt, M. Grätzel, Acc. Chem. Res. 2000, 33(5), 269-277.

[26] N.H. Damrauer, G. Cerullo, A. Yeh, T.R. Boussie, C.V. Shank, J.K. McCusker, Science 1997, 275, 54-57.

[27] C. Bressler, M. Chergui, Chem. Rev. 2004, 104, 1781-1812

[28] A.T. Yeh, C.V. Shank, J.K. McCusker, Science 2000, 289, 935-938.

[29] C. Daul, E. Baerends, P.A. Vernooijs, Inorg. Chem. 1994, 33, 3538-3543; M. Buchs, C. Daul, Chimia 1998, 52, 163 166.

[30] Spectrum measured in a 'dilute crystal' of $\left[\mathrm{Zn}(\mathrm{bpy})_{3}\right] \mathrm{SO}_{4} \cdot 7 \mathrm{H}_{2} \mathrm{O}$ : F. Felix, J. Ferguson, H. G. Güdel, A. Ludi, J. Am. Chem. Soc. 1980, 102(12), 4096-4102.

[31] U. Röhrig, I. Frank, J. Hutter, A. Laio, J. VandeVondele, U. Rothlisberger, ChemPhysChem 2003, 4(11), 1177-1182.

[32] M. Sulpizi, P. Carloni, J. Hutter, U. Rothlisberger, Phys. Chem. Chem. Phys. 2003, 5, 4798.

[33] M. Sulpizi, U. Röhrig, J. Hutter, U. Rothlisberger, Intl. J. Quant. Chem. 2005, 101, 671.

[34] U. Röhrig, L. Guidoni, U. Rothlisberger, ChemPhysChem, in press.

[35] L. Guidoni, U. Rothlisberger, manuscript in preparation.

[36] U. Röhrig, L. Guidoni, U. Rothlisberger, Biochemistry 2002, 41, 10799.

[37] U. Röhrig, L. Guidoni, A. Laio, I. Frank, U. Rothlisberger, J. Am. Chem. Soc. 2004, 126, 15328.

[38] C.S. Page, M. Olivucci, J. Comput . Chem. 2003, 24, 298. 